\title{
Les composés phénoliques des miels : étude préliminaire sur l'identification et la quantification par familles
}

\author{
M.J. Amiot ${ }^{1}$, S. Aubert ${ }^{1}$, M. Gonnet ${ }^{2}$ et M. Tacchini ${ }^{1}$ \\ 1 Laboratoire de Biochimie Métabolique et Technologie. Station de Technologie des Produits Végé- \\ taux \\ 2 Station de Zoologie et d'Apidologie, Institut National de la Recherche Agronomique, Domaine \\ Saint-Paul, 84140 Montfavet, France
}

(reçu le 13-4-1988, accepté le 31-1-1989)

Résumé - Les teneurs en composés phénoliques de huit miels d'origines florales différentes sont très variables. Les miels les plus riches (Arbousier, Chătaignier) renferment les plus faibles proportions d'o-diphénols. Ces substances facilement oxydables seraient responsables de leur coloration brune typique.

L'analyse fine des extraits phénoliques des huit miels par CCM et CLHP/UV (détecteur à barrette de diodes) montre une répartition des composés phénoliques en trois familles : acides benzoïques, acides cinnamiques et flavonoïdes, dont la composition varie avec l'origine florale.

miel — composition chimique — phénol — flavonoïdes — origine florale

Summary - The phenolic compounds in honeys : preliminary study upon identification and family quantlfication. Honeys contain a great number of phenolic compounds; the nature and the quantity of which vary greatly according to the floral origin. Purified phenolic extracts were obtained from eight samples of honey : acacia, Robinia pseudoacacia; strawberry tree, Arbutus unedo; sweet chestnut, Castanea sativa; rape, Brassica napus var oleifera; lavender, Lavandula hybrida; ivy, Hedera helix; fir, Abies pectinata; and sunflower, Helianthus annuus. Honey purity was determined by pollinic, taste, physical and chemical analyses (unpublished data). Acacia, rape, lavender, sunflower and fir were unifloral honeys according to the French Conventional Norms. The three other honeys were not pure but had a dominant floral source of strawberry tree, sweet chestnut and ivy.

The total phenolic compounds assayed by colorimetry using the Folin-Ciocalteu reagent, ranged from $5.8 \mathrm{mg}$ for acacia honey to $96 \mathrm{mg} / 100 \mathrm{~g}$ for the strawberry tree honey. The o-diphenols estimated by the Hoepfner-Arnow's method ranged from 1.2 to $9.1 \mathrm{mg} / 100 \mathrm{~g}$, respectively, for the same honeys. The values are expressed in equivalent chlorogenic acid used as standard. The contents were correlated to the more or less brown color of extracts. Thus, the browning reaction due to the preferential oxidation of o-diphenols was more effective in honeys containing a high quantity of total phenolic compounds (strawberry tree and sweet chestnut) and a proportionally low amount of o-diphenols (Table I).

A thorough analysis of the eight extracts by HPTLC and HPLC equipped with a diode array detector was performed (Table II and Figs. 1 and 2). The distribution of three families, benzoic acids $(B)$, cinnamic acids $(C)$ and flavonoid compounds $(F)$, showed great differences among the honeys 
of different floral origin (Fig. 3). Strawberry tree honey contains $78 \%$ B, $21 \% \mathrm{C}$ and $1 \% \mathrm{~F}$; sweet chestnut honey is characterized by a highly significant ratio of cinnamic acids $192 \% C, 5 \% B$ and $3 \%$ F). Flavonoids are most common in the floral honeys (sunflower : $41 \% \mathrm{~F}, 38 \% \mathrm{C}$ and $21 \% \mathrm{~B}$; Acacia : $42 \%$ F, 37\% C and $21 \%$ B).

However, since the honey sources were rarely from one flower sort, these preliminary results do not give a reliable origin for the honey according to its phenolic compound composition.

honey - chemical composition - phenol - flavonold - floral origin

Zusammentassung - Die phenolischen Komponenten des Honigs : Einleitende Studien zur Identiflkation und Quantlfizierung der Familien. Honige enthalten eine große Anzahl phenolischer Komponenten; die Natur und die Qualität variieren entsprechend der floralen Herkunft. Gereinigte phenolische Extrakte wurden von folgenden acht Honigproben angefertigt : Akazie (Robinia pseudoacacia), Erdbeerbaum (Arbutus unedo), Edelkastanie (Castanea sativa), Raps (Brassica napus var. oleifera), Lavendel (Lavandula hybrida); Efeu (Hedera helix), Tanne (Abies pectinata) und Sonnenblume (Helianthus annuus). Die Reinheit der Honige wurde durch Pollenanalyse, sensorielle und physikalisch-chemische Analysen (unveröffentlichte Daten) bestimmt. Akazie, Raps, Lavendel, Sonnenblume und Tanne sind nach den konventionellen französischen Normen monoflorale Honige. Die drei anderen Honige, die keine reinen Honige sind, haben den Erdbeerbaum, die Edelkastanie oder das Efeu als dominante Herkunft.

Die Gesamtheit der durch Kolorimetrie mit dem Reagenz von Folin-Ciocalteu geprüften Komponenten variierte von $5.8 \mathrm{mg}$ bei Akazienhonig bis $96 \mathrm{mg} / 100 \mathrm{~g}$ beim Honig des Erdbeerbaums. Die Orthodiphenole, die nach der Methode von Hoepfner-Arnow bestimmt wurden, variierten von 1.2 bis $9.1 \mathrm{mg} / 100 \mathrm{~g}$ bei denselben Honigen. Diese Werte wurden in Aquivalent Chlorogensäure als Standard angegeben. Der Gehalt an phenolischen Komponenten ist mit der mehr oder weniger braunen Farbe des Extrakts korreliert. Daher ist die Braunfärbung, die eine Folge der bevorzugten Oxydation der orthodiphenolen Strukturen ist, besonders in den Honigen mit hohem Anteil an phenolischen Komponenten (Erdbeerbaum und Edelkastanie) und proportional gerigem Gehalt an Orthodiphenol anzutreffen (Tab. I).

Eine genauere Analyse der acht Extrakte wurde mit HPTLC und HPLC verbunden mit einem Diodendetektor durchgeführt (Tab. II; Abb. 1 und 2). Die Einteilung der phenolischen Komponenten in drei Familien (Benzoesäurederivate : $B$; Zimtsäurederivate : $C$ und Flavonoide : $F$ ) zeigte große Unterschiede zwischen den Honigen verschiedener floraler Ursprünge (Abb. 3). Der Honig des Erdbeerbaums enthält $78 \%$ B, 21\% C und 1\% F; derjenige der Edelkastanie wird durch einen größeren Anteil an Zimtsäurederivaten (92\% C, 5\% B und 3\% F) bezeichnet. Flavonoide kommen bevorzugt in floralen Honigen (Sonnenblume : $41 \%$ F, 38\% C und 21\% B; Akazie : $42 \%$ F, 37\% C und 21\% B).

Da der Honig selten nur von einer Blütensorte stammt, läßt sich aus diesen vorläufigen Ergebnissen nicht ableiten, daß der Ursprung eines Honigs nach seinem Gehalt an phenolischen Komponenten bestimmt werden könnte.

Honig - chemische Zusammensetzung - Phenol - Flavonoide - florale Herkunft

\section{Introduction}

De nombreuses études sont consacrées à la propolis, source importante de composés phénoliques, notamment de flavonoïdes (Ghisalberti, 1979; Walker et Crane, 1987). Une trentaine de composés ont été identifiés, dont des acides phénols, des flavones, flavonols et flava- nones (Vanhaelen et Vanhaelen-Fastre, 1979). Ces substances phénoliques se trouvent dans les sécrétions de bourgeons et exsudats de divers organes des plantes (Villanueva et al., 1970; Scogin, 1979).

Elles possèdent certaines activités biologiques : germicide, anti-inflammatoire (Adzet, 1987). Ainsi, des effets bactério- 
statiques sont-ils attribués à la pinocembrine (flavone) et à la galangine (flavonol) (Villanueva et al., 1970; Pepeljnjak et al., 1985). Ces substances pourraient se retrouver dans les miels (Bogdanov, 1984). Elles peuvent être considérées comme des marqueurs de l'origine florale (Alix et al., 1985; Tomas-Lorente et al., 1986).

Certains phénols participent à l'arôme au même titre que les substances terpéniques caractéristiques de quelques sources végétales (Lavande, Sapin) ou d'autres composés à noyau aromatique d'origine naturelle, tels les acides phénylacétique et benzoïque (Maga, 1983), abondants dans les miels de bruyère (Speer et Montag, 1984). Par ailleurs, ces derniers composés sont parfois utilisés comme additifs alimentaires (Pinella et al., 1966).

D'autre part, certains composés phénoliques sont impliqués dans les qualités organoleptiques des produits (Pierpoint, 1985). Ainsi, Campus et al. (1983) signalent-ils l'amertume particulièrement forte de certains miels d'Arbousier de Sardaigne, liée à la teneur élevée en polyphénols totaux, un rapport plus faible entre la teneur en composés phénoliques totaux et la fraction polymérisable, exprimée en catéchine.

Enfin, les substances phénoliques interviennent, plus ou moins directement, sur la couleur par l'intermédiaire des flavonoïdes susceptibles de contribuer à la coloration jaune (Harborne et Smith, 1978) et d'une manière générale par les composés phénoliques impliqués dans les phénomènes de brunissements enzymatiques ou non.

Dans le présent travail, nous avons cherché à caractériser quelques miels d'origines diverses par leur contenu phénolique global (dosage de polyphénols totaux). II faut préciser le rôle de ce para- mètre sur la qualité, notamment sur la couleur. Des analyses plus fines (CLHP et CCM) doivent permettre d'identifier un certain nombre de substances et de différencier éventuellement les miels étudiés. Cette recherche d'un nouveau moyen de présomption de l'origine végétale est complémentaire des études sur d'autres critères de la valeur alimentaire des produits de la ruche (Louveaux, 1985).

\section{Matériel et Méthodes}

Les échantillons de 8 miels d'origine végétale différente (Acacia, Robinia pseudoacacia; Arbousier, Arbustus unedo; Châtaignier, Castanea sativa; Colza, Brassica napus var oleifera; Lavande, Lavandula hybrida; Lierre, Hedera helix; Sapin, Abies pectinata; et Tournesol, Helianthus annuus) ont été collectés par la Station d'Apiculture du Département de zoologie de Montfavet.

Ces miels, provenant de différentes régions, représentent un large éventail de la production française, à l'exception d'un seul issu principalement d'Arbousier des maquis de Sardaigne. Les analyses polliniques, sensorielles et physico-chimiques ont permis de les caractériser (données non publiées). Les miels d'Acacia, de Colza, de Lavande et de Tournesol sont issus de nectars et parfaitement monofloraux. Le miel de Sapin est un miel de miellat des Vosges. Le miel de Châtaignier est un mélange naturel issu de nectar et de miellat; de même, celui de Lierre est constitué d'un mélange, récolté en fin de saison. De plus, les 8 miels choisis constituent un échantillonnage large de caractéristiques organoleptiques (couleur, flaveur).

\section{Extraction des composés phénoliques}

Elle a été réalisée sur des échantillons de $250 \mathrm{~g}$ de miel.

L'élimination des substances peu polaires, principalement les pigments caroténoïdes jaunes, est effectuée par malaxage du miel dans 2 parties d'éther de pétrole à l'aide d'un Ultraturrax pendant cinq minutes. Après séparation du surnageant, la masse pâteuse est extraite 3 fois à l'aide d'acétate d'éthyle (1 par- 
tie) après adjonction de sulfate d'ammonium $(0,5$ partie d'une solution aqueuse à $40 \%)$ et d'acide $m$-phosphorique $(0,1$ partie d'une solution à $20 \%$ ) (Macheix, 1974). Ces deux additifs, comme le bisulfite de sodium (Wulf et Nagel, 1980), permettent un meilleur relargage des substances polaires extractibles, en évitant l'oxydation.

Les extraits organiques sont réunis, puis séchés sur sulfate de sodium anhydre; le solvant est évaporé sous vide à une température inférieure à $40^{\circ} \mathrm{C}$. Le résidu $s \theta \mathrm{C}$ est repris dans du méthanol (10 ml); l'extrait ainsi purifié est filtré sur Millipore PTFE $0,5 \mu \mathrm{m}$.

\section{Mesures de couleur}

La méthodologie tristimulaire (CIE) a été décrite précédemment (Aubert et Gonnet, 1983; Gonnet et al., 1986). Les mesures directes de couleur sont réalisées sur les extraits purifiés à l'aide d'un appareil automatique Minolta, équipe d'un système réflectométrique de géométrie $\mathrm{d} / \mathrm{l}$, avec une fenêtre d'observation de $8 \mathrm{~mm}$ de diamètre. L'extrait est présenté dans une cuve de $2 \mathrm{~mm}$ d'épaisseur devant la plaquette étalon en céramique blanche.

Les coordonnées chromatiques sont données instantanément dans le système $x, y$ et $Y \%$ (CIE, 1931; Hunter, 1975), transformées en : longueur d'onde dominante $(\mathrm{nm})$, en pureté ou saturation $(\mathrm{p} \%)$ et en luminance ou brillance (Y\%).

\section{Dosages des composés phénoliques}

Deux méthodes colorimétriques sont utilisées : - l'indice de Folin-Ciocalteu évalue l'ensemble des composés phénoliques, réducteurs du réactif phospho-molybdotungstique (Slinkard et Singleton, 1977);

- l'indice d'Hoepfner-Arnow dose classiquement les polyphénols de structure o-diphénolique (Flanzy et Aubert, 1969; Pourrat et al., 1978).

Les résultats sont exprimés en mg d'équivalent acide chlorogénique pour $100 \mathrm{~g}$ de miel.

\section{Séparation et identification des compo-} sés phénoliques par chromatographie sur couche mince

Les composés phénoliques sont séparés sur cellulose (plaques de cellulose $0,1 \mathrm{~mm}$ d'épaisseur, Merck Réf. 5577) en utilisant l'acide acé- tique à $15 \%$ pour la $1^{\text {re }}$ dimension et le mélange benzène-acide acétique-eau (125-72-3; v/v) pour la $2^{\circ}$ (Chu et al., 1973).

L'observation directe des taches en lumière UV et en présence d'ammoniaque localise, plus ou moins spécifiquement, les structures phénoliques. Divers révélateurs sont utilisés (Ribereau-Gayon, 1968). La pulvérisation de p-nitraniline diazotée, puis de bicarbonate de sodium à $15 \%$ dans l'eau, donne des colorations caractéristiques des acides phénols. Le chlorure d'aluminium, à $5 \%$ dans le méthanol, révèle les flavonoïdes, fluorescents en jaune par excitation à $366 \mathrm{~nm}$.

Séparation et identification des composés phénoliques par CLHP et spectrophotométrie UV

Pour cette étude, l'appareil Varian 5500 est connecté à une barrette de photodiodes (Waters 990).

L'ensemble est placé dans une pièce climatisée à $25^{\circ} \mathrm{C}$. La séparation est réalisée par une colonne en phase inverse ChromSpher C18 (Chrompack), (longueur : $2 \times 10 \mathrm{~cm}$; diamètre : $3 \mathrm{~mm}$ et granulométrie $5 \mu \mathrm{m}$ ) avec une phase mobile eau bidistillée/acétonitrile (l'eau est ajustée à pH 2,6 par de l'acide o-phosphorique). Les gradients linéaires d'élution vont successivement : de 0 à $9 \%$ en acétonitrile pendant $12 \mathrm{~min}$, puis $13 \%$ en $8 \mathrm{~min}, 40 \%$ en 20 min et enfin $70 \%$ en $10 \mathrm{~min}$. Le débit est fixé à $0,8 \mathrm{ml} / \mathrm{min}$. La boucle d'injection est de $10 \mu \mathrm{l}$.

Les spectres des composés ainsi élués sont réalisés instantanément de 200 à $400 \mathrm{~nm}$ dans leur mélange d'élution et comparés à ceux d'étalons commerciaux (Extrasynthèse), passés sur la colonne dans des conditions chromatographiques identiques.

\section{Quantification des composés phéno- liques par CLHP}

L'appareil Varian $\mathbf{5 5 0 0}$ muni d'un détecteur UV 200 est couplé à un intégrateur Varian Vista 402. Les composés sont dosés à $280 \mathrm{~nm}$ par étalonnage externe avec :

- l'acide gallique pour les composés de nature benzoïque,

- l'acide caféique pour les acides cinnamiques et

- la naringénine pour les flavonoïdes.

Les résultats sont exprimés en mg équivalent en étalon pour $100 \mathrm{~g}$ de miel. 


\section{Résultats}

Couleur et teneurs en composés phénoliques

Une forte variabilité de la couleur des miels en fonction de leur origine florale a déjà été mise en évidence (Aubert et Gonnet, 1983). Elle est confirmée par les résultats obtenus sur les extraits phénoliques des 8 miels étudiés (Tableau I). Les mesures de couleur donnent des teintes dominantes autour de $575 \mathrm{~nm}$ (jaunebrun). Les miels les plus rougeâtres (Arbousier) correspondent aux plus grandes longueurs d'onde $(590 \mathrm{~nm})$. Les rapports pureté/luminance variant de 0,19 à 5,3 confirment le classement établi précédemment par Aubert et Gonnet (1983), du plus clair (Acacia) au plus foncé (Arbousier). Par ailleurs, il apparaît une corrélation nette entre ce paramètre de couleur (pureté/luminance) et la teneur en phénols totaux $(r=0,935)$ et o-diphénols $(r=0,877$ ). Ainsi, les miels foncés (Châtaignier, Arbousier) sont-ils les plus riches en phénols, confirmant les résultats obtenus par Campus et al. (1983) sur différents miels d'Arbousier. Cependent, si leur teneur en o-diphénols est élevée par rapport aux miels les plus clairs, les pourcentages relatifs de ces o-diphénols sont les plus faibles : $11 \%$ et $9 \%$ pour le Châtaignier et l'Arbousier respectivement. Notons que la fraction de composés odiphénoliques, les plus oxydables, reste faible pour tous les miels étudiés (29\% au maximum pour le miel de Lavande).

Ces premiers résultats soulignent des teneurs très variables en composés phénoliques totaux et o-diphénoliques des miels suivant leurs origines, qui se traduisent par des différences marquées de leur couleur. $\mathrm{Ce}$ résultat établi, nous avons cherché à caractériser les constituants polyphénoliques par des techniques analytiques plus fines (CCM et CLHP).

Tableau I. Coloration et teneurs en composés phénoliques des extraits de miel.

\begin{tabular}{ccccc}
\hline Origine & Acacia Colza Lavande Tournesol Sapin Lierre Châtaignier Arbousier \\
Robinia Brassica Lavandula Helianthus Ables Hedera Castanea Arbutus \\
pseudo- napus var hybrida annuus pectinata helix sativa unedo \\
acacia oleifera
\end{tabular}

Couleur

\begin{tabular}{lccrrrrrr} 
Longueur & 574,0 & 574,0 & 574,5 & 576,0 & 576,0 & 579,5 & 580,2 & 593,7 \\
d'onde & 10,0 & 15,0 & 33,8 & 34,0 & 36,4 & 67,5 & 76,0 & 68,0 \\
$\begin{array}{l}\text { Pureté } \\
\text { Luminance }\end{array}$ & 52,3 & 50,9 & 47,8 & 47,8 & 39,0 & 28,4 & 29,7 & 12,8 \\
$\begin{array}{l}\text { Pureté/ } \\
\text { Luminance }\end{array}$ & 0,19 & 0,29 & 0,71 & 0,71 & 0,93 & 2,38 & 2,60 & 5,30 \\
Phénols totaux & 5,8 & 8,2 & 9,4 & 11,1 & 18,7 & 16,8 & 27,6 & 96,0 \\
o-Diphénols & 1,2 & 2,0 & 2,7 & 2,0 & 3,8 & 2,3 & 3,0 & 9,1 \\
O-Diphénols/ & & & & & & & & \\
Phénols totaux & 0,20 & 0,24 & 0,29 & 0,18 & 0,20 & 0,14 & 0,11 & 0,09 \\
\hline
\end{tabular}


Analyse chromatographique des 8 miels

\section{Aspect qualitatif}

Les extraits phénoliques des 8 miels sont analysés parallèlement par CLHP (Fig. 1) et CCM (Fig. 2). Bien que les profils chromatographiques soient différents, ils peuvent se diviser globalement en trois parties de polarité décroissante (Fig. 1) correspondant à trois types de composés phénoliques :

- les acides benzoïques $\left(C_{6} C_{1}\right)$, de 0 à $15 \mathrm{~min}$,

- les acides cinnamiques $\left(C_{6} C_{3}\right)$, de 20 à 50 min et

- les flavonoïdes $\left(\mathrm{C}_{6} \mathrm{C}_{3} \mathrm{C}_{6}\right)$ de 45 à 55 $\min$.

Le comportement chromatographique, les profils spectraux et les colorations caractéristiques vis-à-vis de réactifs spé- cifiques ( $p$-nitraniline diazotée et chlorure d'aluminium) permettent d'identifier un certain nombre d'entre eux rassemblés dans le Tableau II. Dans le cas des acides-phénols, 6 dérivés benzoïques et 8 dérivés cinnamiques ont été mis en évidence. Pour les 2 types de dérivés, on note une prépondérance des composés monophénoliques, monohydroxybenzoïque et syringique pour les premiers et coumariques, férulique et sinapique pour les derniers pour l'ensemble des miels. Ceci confirme la faible proportion de composés o-diphénoliques observée (Tableau I). II est intéressant de noter que ces phénols présents dans les miels sont sous forme libre. Cependant, de l'acide chlorogénique ou caféoyl-3-quinique a été détecté dans les miels de Colza, Lavande et Tournesol, bien qu'en très faible quantité.

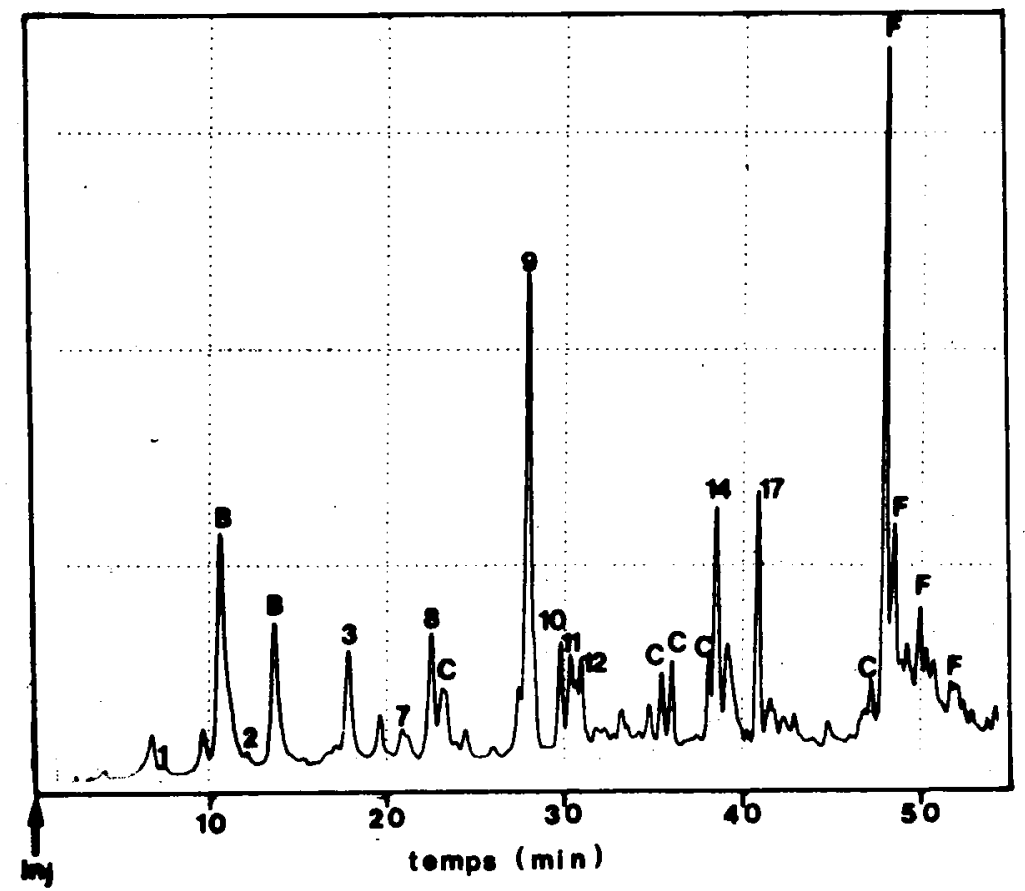

Fig. 1. Séparation des composés phénoliques de miel de Tournesol par CLHP. Les conditions chromatographiques sont décrites dans Matériel et Méthodes. L'identification des composés numérotés est donnée dans le Tableau I. B : acides benzoïques; C : acides cinnamiques; F : flavonoïdes. 


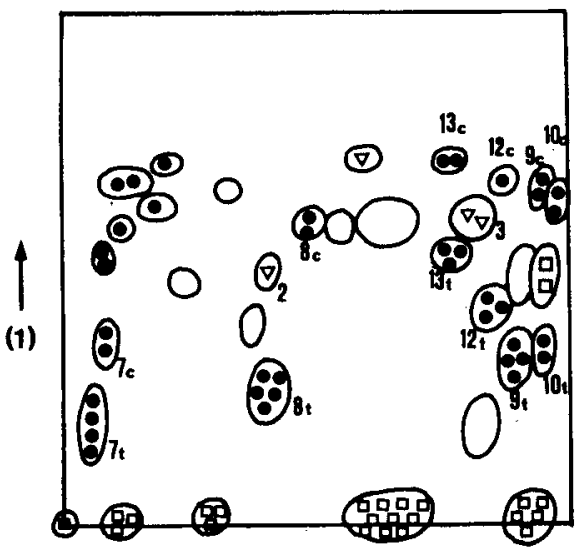

(2)

Fig. 2. Séparation des composés phénoliques de miel de Tournesol sur couche mince. Les conditions chromatographiques sont décrites dans Matériel et Méthodes. (1) et (2) : première et seconde dimension. L'identification des composés numérotés est donnée dans le Tableau $\mathrm{I}$. $\mathrm{c}$ et $\mathrm{t}$ : isomères cis et trans. $\nabla$ : acides benzoïques; - acides cinnamiques; $\square$ : flavonoïdes; pas de symbole : composé non identifié.

Parmi les flavonoïdes, les principales structures rencontrées sont des flavanones, avec des caractéristiques spectrales voisines de la naringénine (Markham, 1982). Elles sont à rapprocher des substances identifiées dans la propolis (Ghisalberti, 1979).

Ce type de molécule phénolique constitue la plus grande partie des flavonoïdes présents dans les miels étudiés. A côté de ces structures ont été détectés des flavones (apigénine) et flavonols (kaempférol et quercétine), notamment dans le miel de Lavande (Tableau II), ainsi que des isoflavones, dont les caractéristiques spectrales se rapprochent de celles de la génistéine (Markham, 1982).

\section{Aspect quantitatif}

Une quantification des principaux phénols (benzoïques, cinnamiques et flavonoïdes) par CLHP a permis de rendre compte de différences importantes entre les miels étudiés (Fig. 3). La classification obtenue est sensiblement différente de celle établie par le dosage des polyphénols totaux et o-diphénols (Tableau I), car seuls ont été dosés par CLHP les composés connus ou dont la structure a pu être rattachée avec certitude à l'un des trois groupes précédemment établis. Néanmoins, les miels d'Arbousier et de Châtaignier restent les plus riches en composés phénoliques et ceux d'Acacia et de Lavande les plus pauvres. Les classements des miels de Colza et de Lierre sont inversés. Dans le premier cas, la structure des pics les plus importants a été élucidée et ces derniers ont donc été comptabilisés alors que pour le Lierre de nombreux pics importants n'ont pu être caractérisés conduisant à une sous-estimation de la

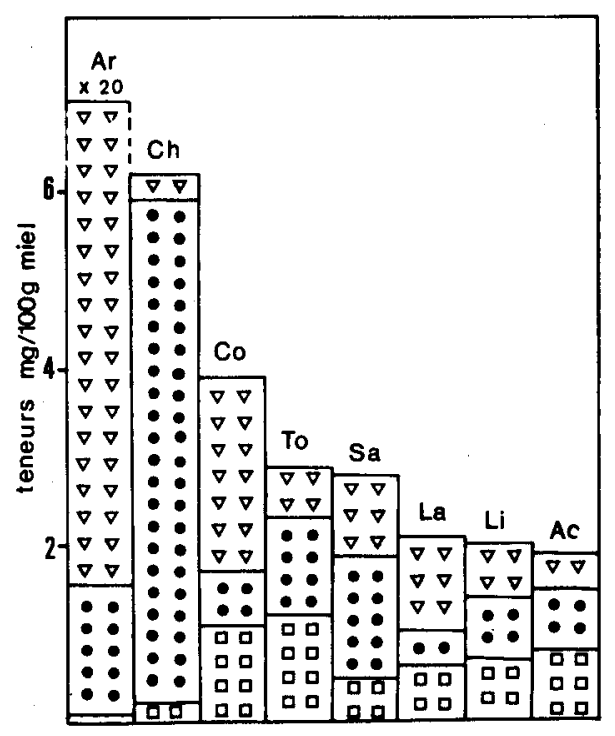

Fig. 3. Composition quantitative en composés phénoliques ( 3 familles) de 8 miels (Ar : arbousier; Ch : châtaignier; Co : colza; To : tournesol; $\mathrm{Sa}$ : sapin; La : lavande; Li : lierre; Ac : acacia). $\nabla$ : acides benzoïques; $\boldsymbol{O}$ : acides cinnamiques; a : flavonoïdes. 
Tableau II. Caractéristiques des composés phénoliques identifiés dans les miels étudiés (Ac: acacia; Ar : arbousier; Ch : châtaignier; Co : colza; La : lavande; Li : lierre; Sa : sapin; To : tournesol). N, numéro de composé; TR, temps de rétention; *, mesuré dans le solvant d'élution; s, épaulement; pNd, après pulvérisation de p-nitraniline diazotée, puis de bicarbonate de sodium; $A$, après pulvérisation de chlorure d'aluminium.

\begin{tabular}{|c|c|c|c|c|c|c|}
\hline$N$ & Composé & $\begin{array}{c}T R \\
\min \end{array}$ & $\underset{n m}{\lambda \max ^{*}}$ & $p N d$ & $A$ & Miels \\
\hline 1 & Gallic & 7,6 & 278 & jaune-brun & - & Ch, La, To \\
\hline 2 & Dihydroxybenzoic & 12,5 & 266,300 & mauve & - & Ch, Co, La, Li, Sa, To \\
\hline 3 & 4-Hydroxybenzoic & 17,2 & 262 & rose & - & Ac, Ar, Co, Sa, To \\
\hline 4 & 3-Hydroxybenzoic & 20,1 & 242,302 & rose & - & Ac, Co, Li \\
\hline 5 & Vanillic & 20,2 & 266,298 & violet & - & $\mathrm{Sa}$ \\
\hline 6 & Syringic & 22,1 & 281 & gris & - & $\mathrm{Ac}, \mathrm{Ar}, \mathrm{Ch}, \mathrm{Co}, \mathrm{Sa}$ \\
\hline 7 & Chlorogenic & 20,8 & $315 s, 333$ & jaune & - & Co, La, To \\
\hline 8 & Caffeic & 22,6 & $305 s, 330$ & beige & - & Ac, Ch, Co, La, Li, Sa, To \\
\hline 9 & P-Coumaric & 28,3 & $300 \mathrm{~s}, 315$ & gris & - & Ac, Ar, Ch, Co, La, Li, Sa, To \\
\hline 10 & Ferulic & 29,4 & $305 s, 330$ & bleu-vert & - & Ar, Ch, Co, La, Li, Sa, To \\
\hline 11 & Sinapic & 29,7 & 242,330 & beige & - & La \\
\hline 12 & M-Coumaric & 30,6 & $284,318 \mathrm{~s}$ & rose & - & Ac, Ch, Co, La, Li, Sa, To \\
\hline 13 & O-Coumaric & 31,4 & $283,331 \mathrm{~s}$ & mauve & - & Ac, Ch, Co, La, Li, Sa, To \\
\hline 14 & Cinnamic & 38,1 & 284 & - & - & Ch, La, To \\
\hline 15 & Rhamnetin & 37,0 & 298,340 & jaune & + & $\mathrm{Ac}, \mathrm{Sa}$ \\
\hline 16 & Kaemperol & 38,3 & 262,377 & jaune & + & Ar, La \\
\hline 17 & Naringenin & 40,8 & 295,340 & jaune & + & Ac, Co, La, Li, Sa, To \\
\hline 18 & Apigenin & 41,3 & 271,344 & jaune & + & La \\
\hline 19 & Quercetin & 41,9 & 268,372 & jaune & + & La \\
\hline
\end{tabular}

teneur globale en composés phénoliques par cette méthode. Nous avons rencontré les mêmes difficultés avec le miel de Sapin, également à dominante de miellat. Lorsque la teneur en polyphénols totaux obtenue par la méthode de Folin et celle fournie par CLHP sont comparées, on peut constater qu'à l'exception du miel d'Arbousier, les valeurs sont systématiquement supérieures à la première. II est 
clair que cette méthode globale donne des valeurs par excès puisque l'ensemble des substances réductrices présentées sont dosées.

Dans le cas du miel d'Arbousier, la plus forte teneur observée en CLHP est probablement due à son exceptionnelle richesse en dérivés benzoïques, monohydroxylés à plus de $50 \%$, exprimés ici en équivalent acide gallique (dérivé trihydroxylé).

Par ailleurs, cette étude chromatographique (Fig. 3) d'une part, confirme l'importante variation des teneurs globales en composés phénoliques des divers miels analysés (Tableau I) et d'autre part, met en évidence une répartition très différente des trois classes. Ainsi, les acides benzoïques varient-ils de moins de $5 \%$ (Châtaignier) à plus de $78 \%$ (Arbousier), les acides cinnamiques de $15 \%$ (Colza) à $92 \%$ (Châtaignier) et les flavonoïdes de moins de $1 \%$ (Arbousier) à $42 \%$ (Acacia). Les miels les plus riches (Arbousier et Châtaignier) ont une composition très différente mais sont tous deux relativement très pauvres en flavonoïdes. Cette classe de composés est beaucoup plus importante dans les miels floraux de Tournesol, Colza, Lavande et Acacia (supérieur à 25\%). Les miels de Lavande et de Colza sont les plus pauvres en acides cinnamiques. Le miel de Lierre présente la composition la plus équilibrée en ces trois classes.

\section{Discussion et Conclusion}

Les observations précédentes sont à interpréter avec des réserves sur les possibilités d'authentification de l'origine des miels par leur composition polyphénolique précise. De plus, l'éventuelle contamination naturelle provenant de la propolis associée aux opercules n'a pas été considérée dans ce travail mais peut être estimée relativement faible et constante pour les 8 miels étudiés. En effet, la principale source de phénols apportés par l'abeille provient des nectars et des sécrétions végétales. Les principales structures identifiées dans les miels sont des acides phénols (benzoïques et cinnamiques), des flavones et flavanones en proportions très variables. Certaines d'entre elles sont à rapprocher de celles trouvées dans la propolis où elles existent essentiellement sous forme libre ou aglycone (Vanhaelen et Vanhaelen-Fastre, 1979). On peut penser que ces dérivés, présents presque exclusivement sous forme estérifiée ou glycosylée dans les végétaux, résulteraient de l'action d'hydrolases apportées par l'abeille.

Par ailleurs, les composés phénoliques des miels sont pour la majorité des monophénols tels que les acides monohydroxybenzoïques, coumarique et férulique. Par contre, les o-diphénols, substrats privilégiés des polyphénoloxydases (Mayer et Harel, 1979; Mayer, 1987) seraient en grande partie oxydés. Les produits d'oxydation formés contribueraient à la coloration brunâtre la plus commune des miels, si on élimine celle apportée par les caroténoïdes non polaires, responsables de la couleur jaune typique des miels de Tournesol par exemple. On peut ainsi noter que le brunissement est particulièrement marqué dans les miels les plus riches en polyphénols totaux et proportionnellement les plus pauvres en o-diphénols.

Afin de caractériser l'origine des miels, une étude plus poussée est nécessaire. Les teneurs globales en composés phénoliques peuvent varier de façon appréciable selon l'origine, l'année et l'environnement des ruches. Ainsi, Campus et al. (1983) obtiennent-ils pour 7 échantillons de miels d'Arbousier des teneurs en polyphénols totaux variant entre 150 et 430 
mg équivalent catéchine. La plupart des sources mellifères ne sont qu'exceptionnellement monoflorales et l'interprétation des chromatogrammes devra être conduite avec prudence.

Des travaux ultérieurs porteront sur la variabilité du contenu phénolique d'un échantillonnage de miels mais aussi de nectars de Tournesol afin de proposer des marqueurs de cette origine florale et éventuellement d'apprécier les modifications apportées par l'abeille. Par ailleurs, la production de miels de Tournesol quantitativement très importante en France pose des problèmes économiques spécifiques (Gonnet, 1984) rendant souhaitable cette étude qui vise à mieux connaître mais aussi à préciser quelques critères de qualité.

\section{Remerclements}

Nous remercions Monsieur R. Borneck, Directeur de I'I.T.API., et sa collaboratrice, Mademoiselle S. Sabatier, Ingénieur Ensbana, pour leur aide matérielle et leurs conseils dans la réalisation de cette étude.

\section{Références}

Adzet T. (1987) Polyphenolic compounds with biological and pharmacological activity. Herbs, Spices, and Medicinal Plants 1, 167-184

Alix J., Grau J., Puigvert A.M. \& De Diego J. (1985) Contribution de la cromatografia en capa fina al analisis del origen floral de las mieles. Vida Apic. 15, 31-33

Aubert S. \& Gonnet M. (1983) Mesure de la couleur des miels. Apidologie 14, 105-118

Bogdanov S. (1984) Characterisation of antibacterial substances in honey. Lebensm. Wiss. Technol. 17, 74-76

Campus R., Madau G. \& Solinas B. (1983) La composizione dei mieli sardi : nota sul contenuto in sostanze azotate $\theta$ polifenoliche. Tecnol. Aliment. 6, 10-15
Chù N.T., Clydesdale F.M. \& Francis F.J. (1973) Isolation and identification of some fluorescent phenolic compounds in cranberries. J. Food Sci. 38, 1038-1042

Flanzy M. \& Aubert S. (1969) Evaluation des composés phénoliques des vins blancs. Ann. Technol. Agric. 18, 27-44

Ghisalberti E.L. (1979) Propolis : a review. Bee World 60, 59-84

Gonnet M. (1984) Un miel de soleils. Rev. Fr. Apic. (434) 483-485

Gonnet M., Aubert S. \& Ferry P. (1986) Evolution de la couleur du miel lors de sa cristallisation. Apidologie 17, 49-62

Häborne J.B. \& Smith D.M. (1978) Anthochlors and other flavonoids as honey guides in the Compositae. Biochem. Syst. Ecol. 6, 287-291

Hunter R.S. (1975) The measurement of appearance. New York, J. Wiley and Sons

Louveaux J. (1985) Le Miel. Cah. Nutr. Diét. 20, 57-70

Macheix J.J. (1974) Les esters hydroxycinnamiques de la pomme : identification, variations au cours de la croissance du fruit et métabolisme. Thèse de doctorat es Sciences, Université Paris VI, $168 \mathrm{p}$.

Maga J.A. (1983) Honey flavor. Lebensm. Wiss. Technol. 16, 65-68

Markham K.R. (1982) Techniques of flavonoid identification. London, Academic Press, 113 p.

Mayer A.M. \& Harel E. (1979) Polyphenol oxidases in plants. Phytochemistry 18, 193-215

Mayer A.M. (1987) Polyphenol oxidases in plants - Recent progress. Phytochemistry 26, $11-20$

Pepeljnjak S., Jalsenjak I. \& Maysinger D. (1985) Flavonoid content in propolis extracts and growth inhibition of Bacillus subtilis. Pharmazio 40, 122-123

Pierpoint W.S. (1985) Phenolics in food and feedstuffs : the pleasures and perils of vegetarianism. In : Annual Proceedings of the Phytochemical Society of Europe, Van Sumere C.F. and Lea P.J. eds, Clarendon Press, Oxford, Vol. 25, 427-451

Pinella S.J., Falco A.D. \& Schwartzman G. (1966) Determination of benzoates and hydroxybenzoates in foods. J. Assoc. Off. Anal. Chem. 49, 829-834

Pourrat H., Jean D. \& Pourrat A. (1978) Purification des polyphénols de l'artichaut par voie enzymatique. Ann. Pharm. Fr. 36, 465-470 
Ribereau-Gayon P. (1968) Les composés phénoliques des végétaux. Ed. Dunod, Paris, $254 \mathrm{p}$.

Scogin R. (1979) Nectar constituents in the genus Fremontia (Sterculiaceae) : sugars, flavonoids and proteins. Bot. Gaz 140, 29-31

Slinkard K. \& Singleton V.L. (1977) Total phenol analysis : automation and comparison with manual methods. Am. J. Enol. Vitic. 28, 49-55

Speer V.K. \& Montag A. (1984) Beitrag zum Vorkommen von Benzoesäure und Phenylessigsäure in Honig. Dtsch. Lebensm. Rundsch. 80, 103-105

Tomas-Lorente F., Tomas-Barberan F.A., Marin F. \& Guzman G. (1986) Nota. Los flavonoides como marcadores quimicos del origen vegetal del polen apicola. Rev. Agroquim. Tecnol. Aliment. 26, 451-454
Vanhaelen M. \& Vanhaelen-Fastre R. (1979) Propolis - II. Identification par chromatographies haute-performance (liquide, gaz-liquide et sur couches minces) des constituants. Bioautographie des chromatogrammes des composés antibactériens. J. Pharm. Belg. 34, 317-328

Villanueva V.R., Barbier M., Gonnet M. \& Lavie P. (1970) Les flavonoïdes de la propolis. Isolement d'une nouvelle substance bactériostatique : la pinocembrine (dihydroxy-5, 7 flavanone). Ann. Inst. Pasteur 118, 84-87

Walker P. \& Crane E. (1987) Constituents of propolis. Apidologie 18, 327-334

Wulf L.W. \& Nagel C.W. (1980) Identification and changes of flavonoids in Merlot and Cabernet Sauvignon wines. J. Food Sci. 45, 479-484 